\title{
Modélisation physique et numérique des interactions sismiques sol-pieu : simulateur de séisme du LCPC
}

\section{N. CHENAF}

\section{J.-L. CHAZELAS}

Laboratoire centra! des ponts et chaussées Division Reconnaissance et Mécaniques des dols

Route de Bonaye BP 4129

44341 Bouguenais Cedex

Nawel.chenafolcpc.f

J-L.chazelas@icpc.ft
1. Nawel Chenaf a reçu le prix Jean Kerisel 2008. décent par le Comité français de mécanigue de sols et de géotechnique (CFMSI), pour les travalux résumés dans cet article. jusqu'au 30 nowembre 2009.

\begin{abstract}
Le travail présenté est une ẻtude expérimentale des interactions inertielle et cinématique, mises en jeu lorsqứ une superstructure fondée sur pieux est sollicitèe par un séisme. A l'aide de modèles réduits centrifugés, trois configurations ont été testées pour observer ces interactions indépendamment puis de manjère combinée. La superstructure qui conditionne l'interaction inertielle a été modélisée par un chevêtre pesant, fixé rigidement en tête du pieu. Le piev a été modélisĕ par un tube creux flexible mis en place par battage. Le massif de sol a été constitué d'un sable de Fontainebleau homogène sec et dense. Cet article présente par la suite une plateforme de modélisation numérique non-linéaire temporelle de la réponse du système superstruclure-pję-sol. Cette plateforme a permis de proposer une première génération de modèles d'éléments d'interface de type Winkler calés sur les résultats expérimentaux. Les modèles d'éléments d'interface ont été choisis dans la littérature parmi ceux fondés sur des paramętres physigues.
\end{abstract}

Mots-clés : interactions sol-pieu, interaction inertielle, interaction cinématique, modèles réduits en centrifugeuse, simulateur de séisme, modèle numérique, modèles de Winkler.

\section{Soil-pile seismic interactions physical and numerical modelling: LCPC earthquake simulator}

The present work is an experimental study of the inertial and sinematic interactions for a superstructure-pile-soil systern submitted to an earthquake, based an reduced scale model tests in the centrifuge. Three configurations were set up in order to observe each interaction separately and knen to combine them. The superstructure generating the inertial effect was modelled by a heaw pile cap attached on the plie head. The flexible pile was driven into the sol. The sol deposit was a homogenous dry Fontainebleau sand layer. In a second part. a numerical code is presented to compute the temporal non-linear response of superstructure-jile-soil system. The mumerical model is based on the $1 \mathrm{D}$ Winkler beam on non-linear bearings and computes khe response in the time domain. The thfferent inter'ace elements based on physical parameters were chosen from the literature and fitted on the experimental data.

Key words: sol-pile interactions, inertial interaction, kinemakic interaction, scale centrifuge tests, earthquake simulator, numerical model, Winkler model. 


\section{Introduction}

Lorsqu'une structure fondée sur pieux subit ur séisme, les pieux interagissent avec le sol par deux mécanismes : l'interaction inertielle et l'interaction cinématique. Linteraction cinématique est le chargement du pieu par le mouvenzent du sol dü à la propagation des ondes sismiques. Linteraction inertielle est due aux forces d'entrainement, exercées par la superstructure sur le systène pieu-\$ol, engendrées par l'accélération de la masse du chevétre, due à l'effet cinématique, L'approche rationnelle de l'étude puis de la modélisation de l'interaction sismique sol-pieu qui combine les deux effets suppose de les étudier séparément puis d'étudier leur superposition. On sait que la séparation des deux effets ne sera pas une sinple addition compte tenu de la non-linéarité du comportement du sol, même si Mylonakis et at. (1997) proposent de l'envisager pour des systemes modérément non linéaires. Largument en faveur d'une telle dissociation et addition simple est que la déformation d'un pieu, soumis à une charge latérale transmise par la superstructure, s'attênue rapictement avec la profondeur, les déformations clues à l'effet inertiel ne sont donc significatives qu'en surface, alors que les ondes sismiques m'induisent d'importants déplacements qu'en profondeur. La superposition des deux effets peut donc être une approximation raisonna* ble si la non-linéarité du sol est explorée.

Dans des cas plus complexes, caractérisés par de fortes non-linéarités, il est possible dobserver ces deux interactions indépendamment l'une de l'autre : puisque l’interaction inertielle est due ta la masse de la superstructure, 1'interaction cinérnatique sol-pieu peut être isolée à partir de la réponse d'un pieu sans superstructure sollicité par des ondes sismiques. Au contraire, la sollicitation d'un piel. avec une stiperstructure par un impact en tête pernel d'observer la réponse inetielle du système superstructure-pieu-sol. Enfin, un essai sisnique appliqué à un ensembje complel superstructure=pieu-sol met en jeu les deux interactions. C'est l'ensemble de ces trois approches expérimentales qui est présenté dans cet article.

Les travaux de modelisation des deux interactions séparées et combinées sont nombleux. Les approches analytiques de l'interaction cinématique (Margason. 1975 ; Hores-Berrones et Whitman, 198 ? ; Dobry et O'Rourke, 1983; Barghouti, 1984; Gazetas, 1984 : Souloumiac, 1986 ; Makris et Gazetas, 1992 ; Shahrour et al., 1994 ; Nikolaou et al., 2001\} et de l'interaction inertielle LNovak, 1974 ; Kavnia et KauseL, 1982 ; Matlock et al., 1978; Novak et Aboul-Ella, 1978 ; Nogami, 1991 ; El Naggar et' Novak, 1996 ; Boulanger ef al, 1999) sont faciles à mettre en cuve et prennent en compte, selon l'approche, divers paramètres d'interaction et les caractéristiques du sol, du pieu et de l'excitation sismicue. Elles ont cependant été développées dans le domaine fréquentiel ou pour un conportenent linéaire ou liréaire équivalent, ce qui en limite l'usage, surtout dans une perspective de superposition des deux interactions.

Les approches numériques basées sur des nodèles aux élements finis ont principalement étudié le comportement du système couplé superstructure-pieusol soumis à un séisme (El Naggar et Bentley, 2000; Mahesliwari et al., 2004a et 2004bi. Les analyses consacrées à l'interaction cinématique se sont plutôt intéressées à l'étucle de l'effet de cette interaction sur le déplacement de lá tête ciu piè, ce qui est loçique par rapport à son rơle comme générateur de l'accélération des superstructures. Nikolaou et al. (2001) ont signalé cependant, cians un rapport de retour d'experience. que des ruptures de pieux ont été localisées dans la zone proche du pied du pieu sans que la liquéfaction ne soit mise en cause. L'évaluation de cet effet sur toute la hauteur du pieu s'avère donc déterminante.

La modélisation physique a aussi traité du chargement sismique plus que du chargement en tête (Meymand, 1998; Wilson, 1998 ; Tokimatsu et al., 2005), l'effet inertiel pur a souvent été déduit d'essaj sismiqué par une décomposition analytique des deux effets [Tokimatsu et al., 2005]. I] convient également de rappeler l'existence de l'essai STATNAMIC (E)-Naggar. 1998 : Chakraborty, 2000l cui consiste à appliquer un choc en tếte d"un pleu en vraje grandeur. Cet essai foumit un moyen de produire de grands chargements axiaux et latéraux sur des fondations par le biais d'une masse de réaction mobile. L'énergle développée lors de l'inpact est entierement restituée dans une bande de fréquences proche de la fréguence fondamentale du systène sol-piet. engendrant ainsi des forces d'inertie signifi. catives, ce qui a permis de déterminer les propriétés dymaniques de l'interaction sol-pieu.

Dans le domaine des essais en modèles réduits, Grundhoff et al. (1998) ont commencé une telle étude à l'ajde d'un simulateur d'impact en tête du pieu çui fonctionne en microgravité, leurs résultats ne sont pas connus. Bonab (2003) a repris ce concept pour concevoir un générateur de chocs répétitifs en vol sans arrèter la centrifugeuse et a présenté une étude paramétrique expérimentale de l'interaction inertielle.

Le travail présenté ici a complété cette étude para. métrique en utilisant le même outil de chargement, qui ne serg rappelé ici que succinctement. En revanche, le présent article dêtaile plus particuliêrement les experimentations sous sollicitations sismiques en centrifugeuse qui constituent une prenière en France. Les résultats expérimentaux en terme d'évolution temporalle des moments fléchissants en fonction de la profondeur seront présentés et commentés pour les trols configurations d'essai et donc pour les trois niveaux d’interaction i inertielle, cinénatique et combinée.

Parallèlement aux travaux expérimentaux, des outils numérigues d"interprétation des données ont été développés. Il s’agit d’une plateforme numériçue fondée sur le modéle de la poutre de Winkler sur appuis non linéaires dans le domaine temporel. Cette platefome vise à permettre la constitution et le test d'éléments d'interface représentatifs des deux formes d'interaction. Cet article présente cette plateforme et sa validation dans le domaine statique par comparaison avec différents outils : solution analytiçue, conparaison. avec PIIATE-LCPC (Baguelin et al., 1976) et compa. raison avec des résultats expérimentaux. Une vérification de la plateforme dans le domaine dynamique sera aussi présentée à travers un exemple d’ajustement d'un modèle d"interaction inertielle sur les résultats expérimentaux.

\section{2}

\section{Modélisation physique à échelle réduite de l'interaction sol-pieu}

Dans cette étude expérimentale, deux types de chargement sont utilisés pour simuler les différentes interactions auxquelles est sujet le système sol-pieu 
sous charges dynamiques : des essais d'impact en tête du pieu pour simuler en premier lieu l'interaction inertielle pure et des essais sismiques avec le Simulateur de Séisme du LCPC pour modéliser l'effet cinématique pur et les deux interactions conbinées. Le sol, le pieu modèle, le chevêtre qui écuipe la tête du pieu ainsi que l'instrumentation mis en ceuvre sont identiques pour les deux types de chargement et pour les trois configurations d'essal. Cette partie résume les procédures expérimentales établies ainsi que les moyens mis en cuvre pour réaliser les essais.

\section{1}

\section{Procédures expérimentales}

\section{Propriétés du sol}

Le massif de sol, reconstitué par pluviation dans des conteneurs rigides, est un sable de Fontainebleau homogène, fin, sec et dense (1 $600 \mathrm{~kg} / \mathrm{m}^{*}$; la densité relative Dr est égale à $86 \%$ ). Le tableau I résume les principales caractéristiques du sable utilisê.

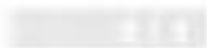

\section{Propriétés des pieux modèles}

Les pieux modèles ont été conçus pour un facteur d'échelle sur les longueurs de $1 / 40^{\circ}$ et donc des essais à $40 \mathrm{~g}$ (facteur d'échelle $\mathrm{N}=40$ ). Les caractéristiques géométriques et mécaniques du pieu prototype modélisé et celles du pieu modéle sont résumées dans le tableau Il.

Le pieu modèle est souple, sa longueur de transfert a été estimée à 3 m (Remaud, 1999).

Le pieu modèle est battu dans le massif de sable à $1 \mathrm{~g}$. a l'aide d'un dispositif simple de battage conçu a L LCPC (Fig. 1]. Ce dispositif assure une bonne répétitivité du battage d"un essaì un autre.

Le pieu modèle est équipé de vingt niveaux de mesures de déformation. Chaque niveau de jauge est constitué de deux jaugas collees en regard sur les deux génératrices du pieu. Les jauges sont collées par paire

\begin{tabular}{|c|c|c|c|c|}
\hline TAB & \multicolumn{4}{|c|}{$\begin{array}{l}\text { Propriétés physiques du sable de } \\
\text { Fontainebleau. } \\
\text { Physical properties of the Fontainebleau sand. }\end{array}$} \\
\hline \multicolumn{3}{|c|}{ Densité $\left(\mathrm{kg} / \mathrm{m}^{3}\right)$} & \multicolumn{2}{|c|}{ Indice des vides } \\
\hline $\mathrm{P}_{5}$ & $P_{\text {di may }}$ & Prd mun & $\mathrm{e}_{\max }$ & $e_{m \mid \pi}$ \\
\hline 2650 & 1676 & 1412 & 0.887 & 0,581 \\
\hline
\end{tabular}

et montées en demi-pont de manière à permettre l'évaluation directe de la déformation de flexion à chacue étage de paire de jauges. Les paires de jauges sont rëparties sur la fiche du pieu tous les $15 \mathrm{~mm}$ sur une longueur totale de $285 \mathrm{~mm}$. Les jauges sont protégées par une couche de gelcot.
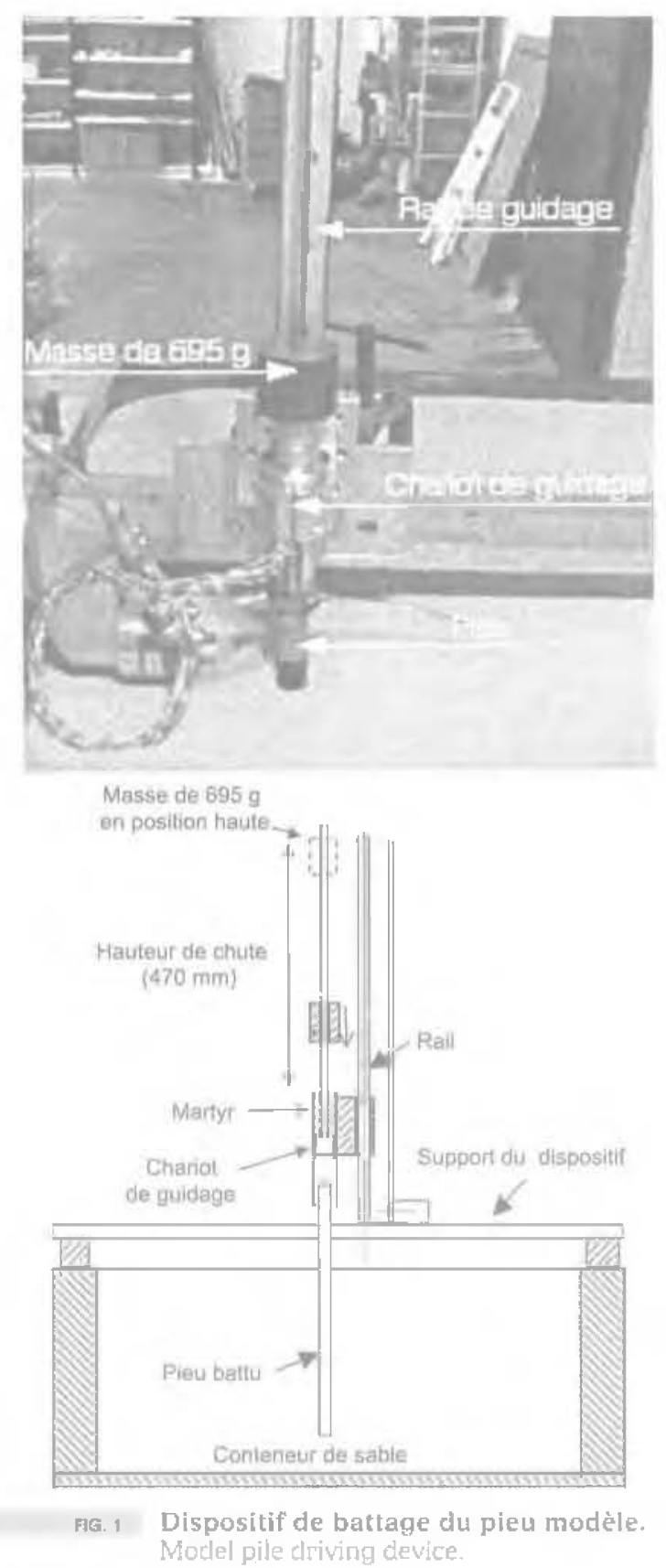

TABLEal II Caractéristiques mécaniques et géométriques du pieu prototype et du pieu modète. Prototype and model piles mechanical and geometrical charadteristics.

\begin{tabular}{|c|c|c|c|}
\hline & Facteur d"êchelle & Pieu prototype & Pleu modèle \\
\hline Matériau & & Acier & Aluminum \\
\hline Longueur [m] & $\mathrm{N}$ & 15.2 & 0.380 \\
\hline Longueur de fiche $\mathrm{D}$ [m] & N & 12 & 0,3 \\
\hline Diamètre B [m] & $N$ & 0.72 & 0,018 \\
\hline Diamêtre b [n] & $\mathbb{N}$ & 0,685 & 0,015 \\
\hline Module dYoung du matériau $\mathrm{E}_{p}[\mathrm{MPa}]$ & & $2.10^{k}$ & $7,4.10^{4}$ \\
\hline Moment d'inertie de la section $I_{p}\left[\mathrm{~m}^{4}\right]$ & $\mathrm{N}^{-1}$ & $2.38 \cdot 10^{-4}$ & $2,67.10^{14}$ \\
\hline Rigidité $\mathrm{I}_{\mathrm{i}}\left[\mathrm{MN}, \mathrm{m}^{2}\right]$ & $\mathrm{N}^{9}$ & 476 & $197.10^{-1 r}$ \\
\hline
\end{tabular}




\section{Le chevêtre}

Une masse pesante est fixée en tête du pleu pour simulel l'effet inertiel. Dans le cas des essais de choc, il permet aussi de protéger la tête du pieu d'un éventuel endommagement, puisque le générateur de choc est dirigé vers le centre de gravite de la tête du pieu qui correspond à l'extrénité du pieu. Le chevêtre a étẻ conçu pour assurer une liaison rigide avec la tête du pieu, pour éviter une discontinuité de la rotation ainsi que des vibrations locales des pièces. Le chevêtre en aluninium modèle $\left(30 \times 50 \times 50 \mathrm{~mm}^{3}\right)$ pesant $188,3 \mathrm{gr}$ simule une superstructure prototype de $13 \mathrm{t}$

L'accélération au niveau du chevêtre est mesurée par un accéléromètre collé à hauteur de son centre de gravité et sur la face opposée à celle qui reçoit l'impact. Le déplacement du chevêtre est enregistré par un capteur laser analogique. Ce capteur est fixé sur un support métallique de telle sorte que le spot lumineux du laser soit dirgé vers le centre de gravité du chevêtre.

\section{Dispositif et séquences de chargement}

Le premier type d'essai, qui visait à simuler exclusivement l'interaction superstructure-sol-pieu inertielle, a été mis en place par Bonab [2003). Cet essai dit a inertiel n repose sur la génération d'impact en tête du pieu par un générateur d'impact, constitué d'une bobine électromagnétique qui accélère une bille (Fig. 2). Le choc est géré depuis la salle de commande de la centrifugeuse. Le dispositif permet d'appliquer aussi bien un choc qu'une séquence de chocs d'amplitude constante ou non. L'essas inejtiel mis en place est schématise sur la figure 3.

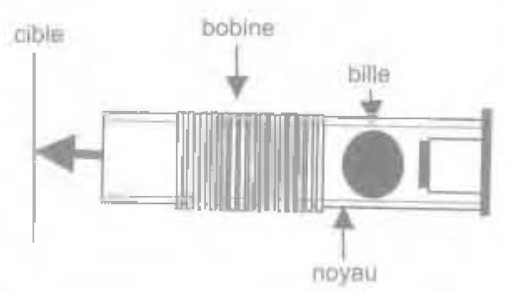

Fo \& Schéma du générateur d'impact ęn tête du piet. Inertial Jading device.

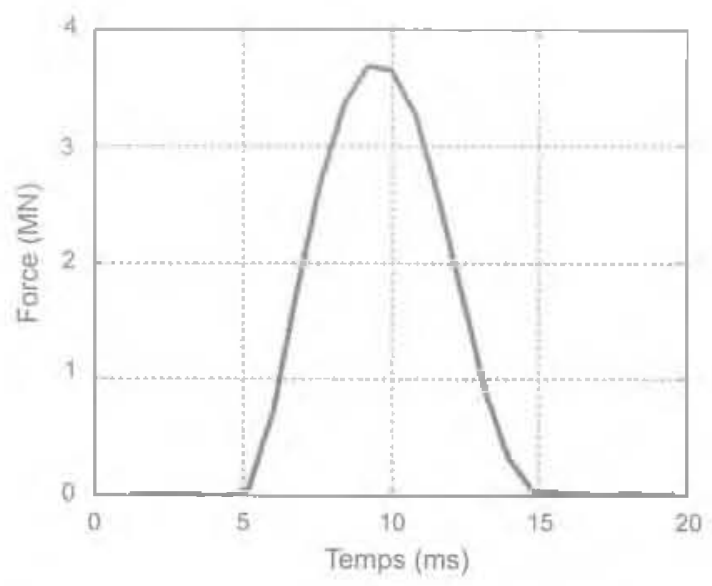

(a)
La force dimpact est enregistrée par un capteur de force vissé sur le chevêtre, un matériau de contact est collé sur la face du capteur qui reçoit le choc, pour le protéger et pour prolonger la durée de l'impact.

Lenregistrement temporel, ainsi que le contenu fréquentiel, de la force appliquée sur le chevêtre lors de l'essai inertiel sont donnés sur la figure 4 . Considérant que la fréquence propre du système pieu-sol constaté est de l'ordre de 3,6 Hz prototype [Chenaf, 2007], soit une période propre de $0,27 \mathrm{~s}$, la mesure de la force montre que l'impact appliqué est très court et peut être considéré comme un Dirac de force.

Les essais sismiques. qui ont permis de simuler l"interaction sol-pieu cinématique et la combiraison des deux interactions, étaient les premiers essais réalisés avec le Simulateur de Séisme (SdS) dont s'est doté le LCPC. Le SdS est une table vibrante embarquée dans la nacelle de la centrifugeuse. Ainsi, au cours de la rotation de la centrifugeuse, le modèle est soumis simultanément à un champ de macrogravité engendré par I’accélération centrifuge, selon la verticale cu modèle. et à une accélération sismique horizontale par rapport au modèle. Ce dispositif développé par l'entreprise Actidyn Système a déjà été largement présenté (Derkx et aI., 2006a et 2006b). Ses principales caractéristiques sont rappelées dans le tableau III.

Le SdS est un dispositif électro-hydraulique qui permet de commander des séismes sinus d'une durée maximale d'une seconde dans la bande 20-200 Iz pour la pleine charge ou des séismes large bande tels que
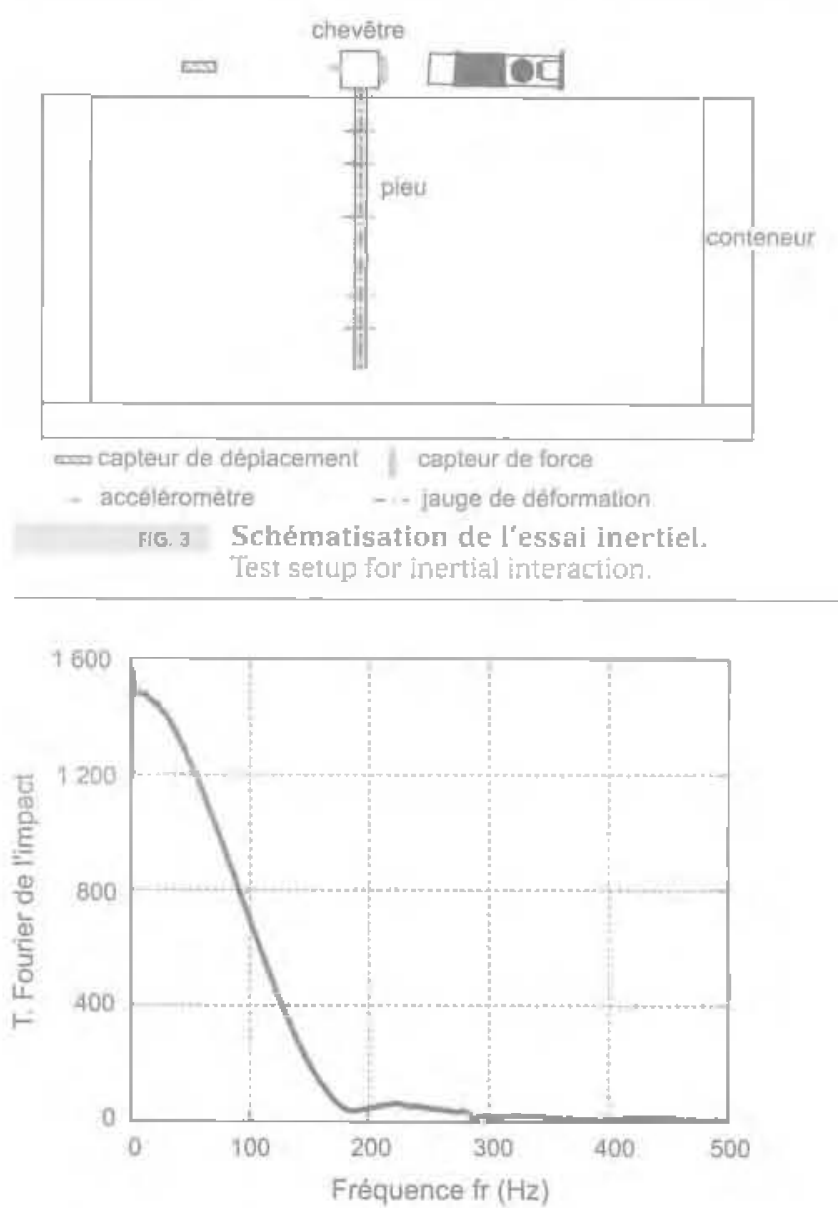

(b)

FIG A Enregistrement temporel (a) et fréquentiel (b) de l'impacı en tête du pieu. (a) Time record of the horizontal force appled at the pile cap for the inertial tests (b) FFT of this force. 
TABLEaU ut Performance du simulateur de seisme du LCPC.

ICPC earthquake sinulator performance.

\section{Modẻle}

$400 \mathrm{~kg}$
Prototype (réel)

Masse utike dimension prototype dass le contenew

$32 \times 24 \times 22 \mathrm{~m}$ au $1 / 40$

$64 \times 28 \times 44 \mathrm{~m}$ aw $1 / 90$

\begin{tabular}{|c|c|c|}
\hline Champ de gravité centrifuge & 10 à $80 \mathrm{~g}$ & $1 \mathrm{~g}$ \\
\hline Déplacentent maximal & $5 \mathrm{~mm}$ & $\begin{array}{l}20 \mathrm{~cm} \text { : } 1 / 40 \\
40 \mathrm{~cm} \text {. } 1 / 80\end{array}$ \\
\hline Witesse maximale & $1 \mathrm{TH} / \mathrm{s}$ & $1 \mathrm{~m} / \mathrm{s}$ \\
\hline Accélération maximale & $20 \mathrm{~g}$ à $1 / 4040 \mathrm{~g}$ a $1 / 1 / 80$ & $0,5 \mathrm{~g}$ \\
\hline Bande de fréguence en sinus & $20-200 \mathrm{~Hz}$ & $\begin{array}{c}0.5-5 \mathrm{~Hz} \text { au } 1 / 40 \\
0.25-2.5 \mathrm{~Hz} \text { au } 1 / 80\end{array}$ \\
\hline Durée maximale du séisme siruss & 15 & $\begin{array}{l}40 \mathrm{~s} \text { au } 1 / 40 \\
80 \mathrm{~s} \text { au } 1 / 80\end{array}$ \\
\hline
\end{tabular}

des enregistrements de séismes rếels ou des séismes synthétiques.

Lors des essais de réception, il a été démontré que cette machine disposait de la puissance nécessaire pour réaliser le programme spécifié, en revanche les mouvements appliqués à la masse d'essai présentaient trop de mouvements parasites (er lacet et en basculement). Cependant, des annéliorations réalisées en janvier 2007 ont permis d'obtenir un respect parfaitement conforme aux spécifications en sinus et en séisme réel pour une masse légèrement réduite et à $40 \mathrm{~g}$. Il reste encore à vérifier l'amêlioration complête des spécifications.

La figure 5 présente le SdS du LCPC disposẻ dans la nacelle de la centrifugeuse ainsi qu'un schéma descriptif de sa mêcanique.

Comme il est indiqué sur la figure 6, le signał sismique choisi pour les essais présentés par la suite correspond à une fonction harmonique de type sinusoidale. Ces principales caractéristiques sont données sur cette figure à l'échelie du prototype. Dans le tableau IV, ces caractéristiques sont données avec leur équivalence à l'échelle du modèle.

Lors des essais sismiques, en plus de l'instrumentation spécifique au pieu et au chevêtre (cf. $\$ 2.1 .2$ et 2.1.3j, des accélêrornètres ont étè disposés dans le so en diffêrentes profondeurs en cours de pluviation. Ces capteurs ont permis le suivi de l'êvolution du déplace. ment du sol en cours d'essai. Ainsi que son évolution
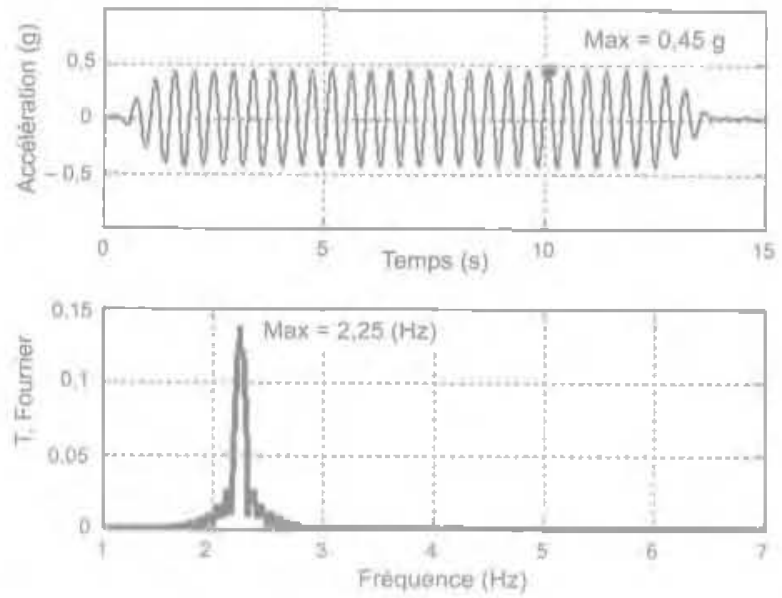

FGG a Caractéristiques de t'accélération du séisme simulé.

Simulated earthquake characteristics fup in the bime domain. lower in the frequency domaini.

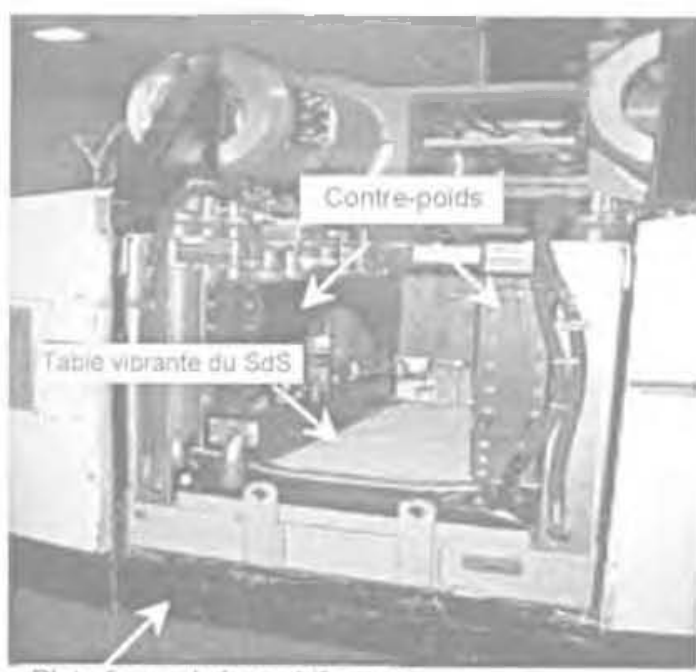

Plate-forme de la centrifugeuse

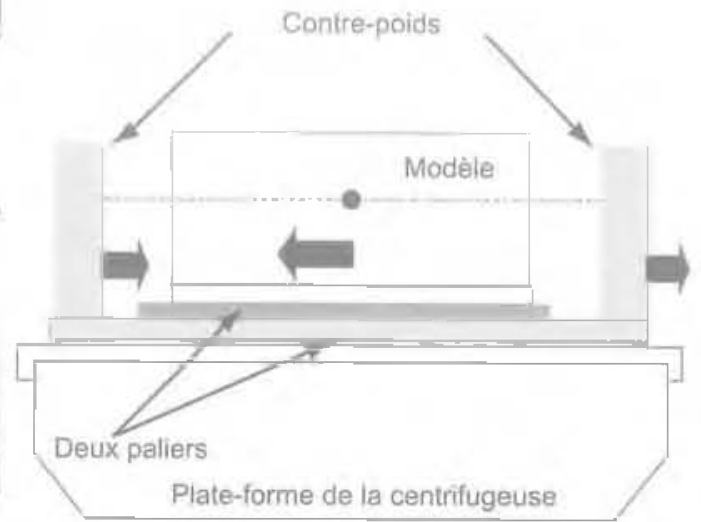

Plate-forme de la centrifugeuse

FG : Photo du simulateur de séisme du LCPC disposé dans la centrifugeuse. Earthequake simulator at LCPC sean embarqued in the centrifige basket "The front counterweight is withdraw. 
Tanten \% Caractéristiques modèle et prototype du signal sismique simulé.

Model and prototype characteristics of simulated seismic signa!

\begin{tabular}{lcc} 
& Modele (1/40) & Prototype \\
\hline Amplitude [g] & 18 & 0,45 \\
Amplitude [m/5] & 176,5 & 4,4 \\
Durea [s] & 0,3 & 12 \\
Nbre d'alternance [.] & 30 & 30
\end{tabular}

d'un essai sismique à un autre, puisque trois configurations d'essai sismique ont été mises en place : un essai sisnaique sur un massif de sol visait la caractérisation du déplacement du sol en u champ libre w, cest-ändire sans l'effet de la présence du pieu, la seconde configuration visait à simuler l'interaction cinématique, le pieu dans ce cas n'a pas été équipé du chevétre en tête, la dernière a pris en compte tout le système chevêtrepieu et sol oủ il s'agissait de simuler les deux effets combinés. La figure 7 schématise cette demière configuration.

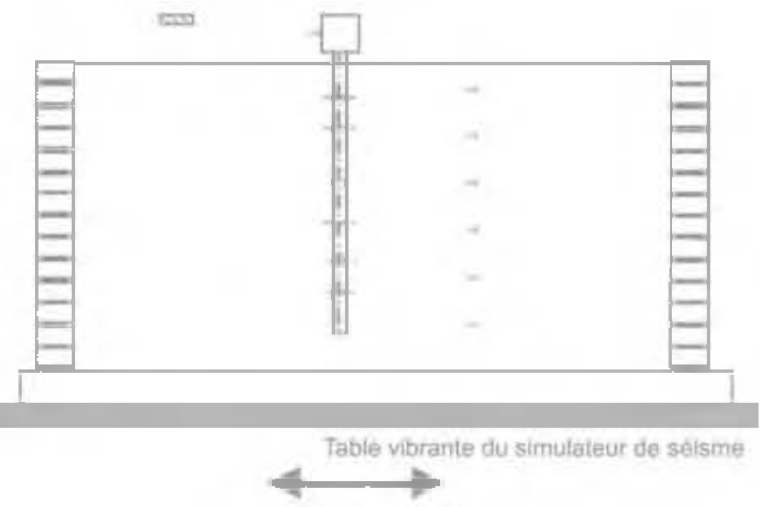

Signal sismique d'entrée

Fig. 7 Dispositions experimentales de l'essai sismique sur l'ensemble chevêtre-pieu-sol. Test setup for combined interactions lfor kinenatic interaction the cap is withdraw'

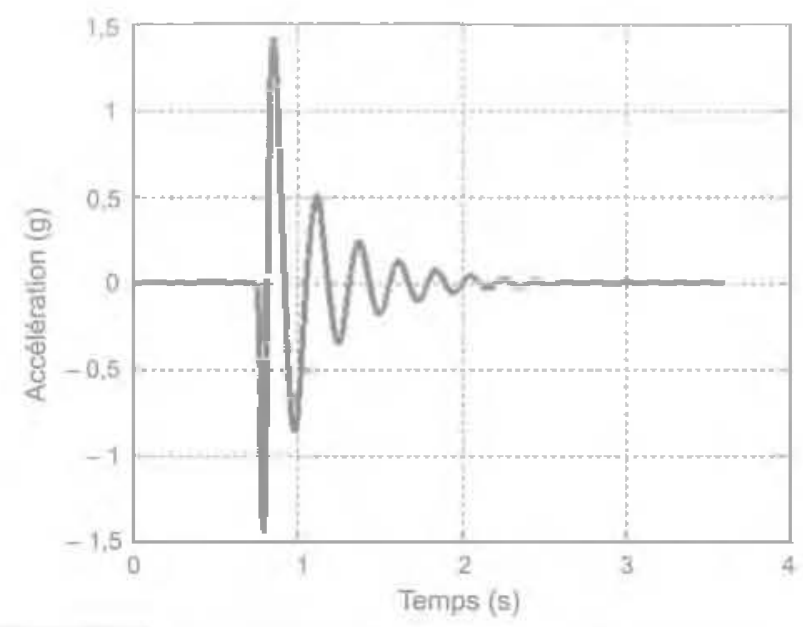

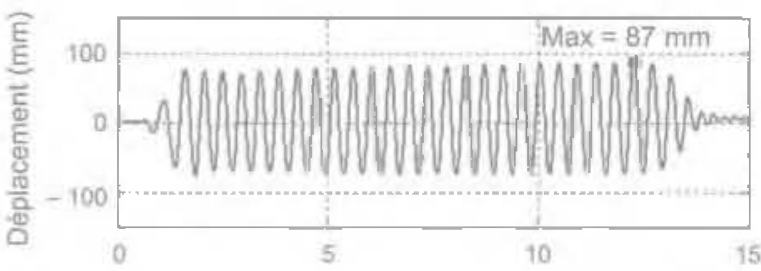

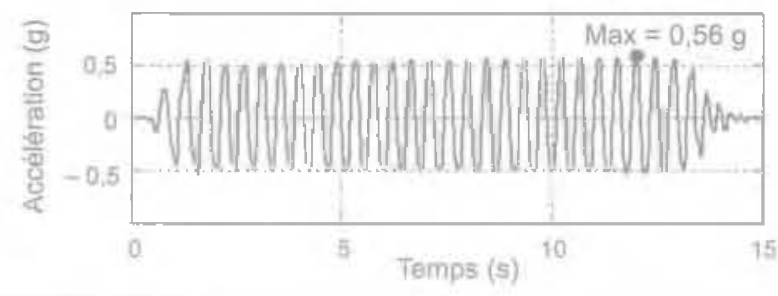

Fig. 9 Déplacement et accélëration du chevểtre : Essał sismintue.

Cap displacement and acceleration: seismic test.

\section{9}

\section{Mesures acquises lors des essais inertiels et sismiques}

\section{Accélération - Déplacement du chevêtre}

L'accélération du chevêtre ainsi que le déplacement correspondant, mesuré par le capteur laser, sont donnés sur la tigure 8. Ces deux enregistrements corres. pondent à un mouvement oscillatoire amorti, quil s'atténue après 2 s. La figure 9 représente le déplacenent et I'accélération mesurés en tête du pieu lors d'un essai sismique, réalisế avec un pieu équipé du chevêtre. Le mouvement est harmonique forcé [non-amorti]. [] comrespond au signa! sismique d"entrée en fréquence et en nombre d'alternance (Fig. 6). Les mesures de déplacement et d'accêlération en tête du pieu permettent d’uné part de vérifier la réponse instantanée du pieu à la sollicitation, et d'autre part sont utilisées comme conditions aux limites lors de l'établissement des boucles p-y (Chenaf, 2007), pétant la réaction du sol et y le déplacement du pieu.

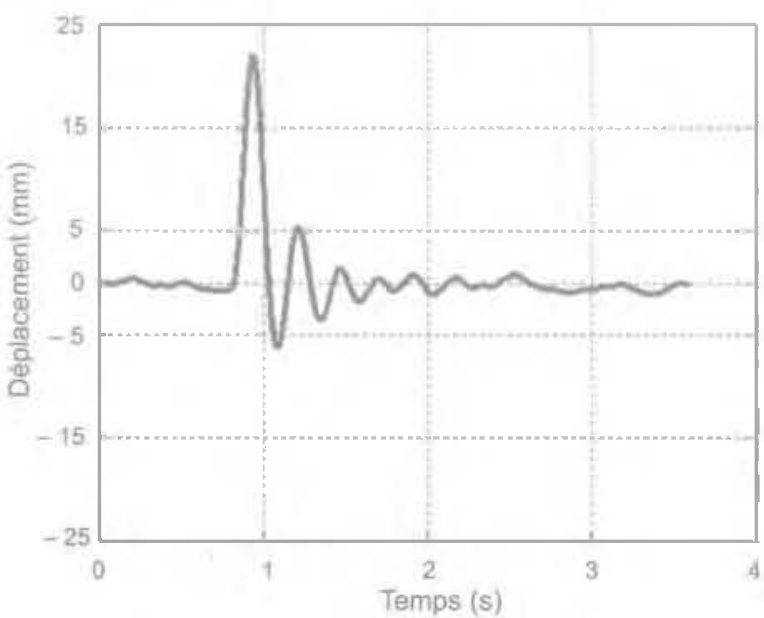

FIG A Accélération et déplacement du chevêtre : essai inertiel.

Cap acceleration and displacentint: lnertial test. 


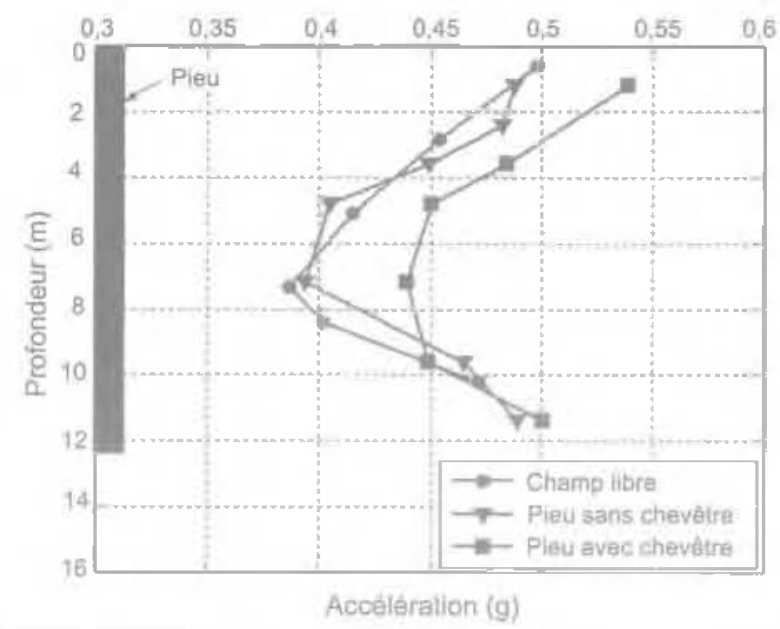

FiG. so Comparaison des accélêrations maximales du sol pour trois configurations d'essaí sismique.

Compatisonofthemaximumacceleration profiles in the three selmic tests configurations.

\section{Accélération de là colonne de sol}

Les essais sismiques réalisés dans cette étude sont particuliers, la notion de déformation dans le champ proche du pieu et dans le champ lointain n"est pas applicable. Le massif de sol a été reconstitué dans un conteneur rigide dont la base est solidaire du simulateur de seisme.

Afin de caractériser la réponse de la colonne de sol dans ces conditions, les accélérations maximales du sol issues de l'essai sur le massif de sol en champ libre ont été comparées à celles déduites des essais sur pieu équipé ou non équipé du chevêtre. Cette cornparaison est représentée sur la figure 10. Les accélérations comparées sont les accélérations horizontales mestrees dans le sol à environ 5 diamètres du pieu. Notons que les accélérations du massif de sable dans le cas des chocs en tête n'ont pas êtể mesurées.

La figure 10 montre que ís courbe d'accélération du sol en "champ libre y est comparable à celle de l"essai sur pieu non-équipé du chevêtre. Le mouvement du sol n'est donc pas affecté par la présence du pieu. On en déduit que le pieu subit un effet cinématique pur dû́ á un mouvement d'entrainement par le sol.

La courbe de l'essai sur pieu équipé du chevếtre a la même allure que les deux courbes précédentes jusqu'à $7 \mathrm{~m}$. L'ecart en surface entre les courbes est quasi-constant. Ati-delà de 9 m de profondeur, les courbes sont confondues. L'écart d'amplitude en surface est dû à l'effet inertiel du chevêtre ; en pied du pleu, cet effet disparait.

\section{Moments fléchissants}

L'étalonnage du pieu instrumenté par essais statiques en flexion permet de convertir directement les informa. tions des paires de jauges en moment à chaque niveau. I! est donc possible de tracer les profils des moments fléchissants en fonction de la profondeu" et au cours du temps. Les figures 11, 12 et $\uparrow 3$ présentent ces profils pour les trois configurations d'essai. Pour la lisibilité des figures, sont présentés des profils à différents instants formant une sèquence du mouvement. La flèche dessinée sur le graphe indique le sens du mouvement.

En s'appuyant sur" l"evolution temporelle de ces profils, il est possible de tracer l"enveloppe des moments maximaux à chaque profondeur au cours du temps pour chaque configuration ainsl que la zone au-delà de laquelle les moments peuvent ètre considérés comme กuls.

Dans le cas de l'essai inertiel [Fjg. 11), le moment maximum est estimé à $1100 \mathrm{kN}$. m et se situe à une profondeur égale à $1,8 \mathrm{~m}$ par rapport à la surface libre du sol. Ce moment dépend bien sur de l'anplitude de la force appliquée. Les noments sont négligeables a delà dè 6 ith.

Sur la figure 12, les moments obtenus de l'essai d'interaction cinématique pure croissent de la surface jusqu'à 6,6 m et décroissent par la suite jusqu'en pled du pieu. Le moment maximum estégal à $223 \mathrm{kN}$.m.

Les moments fléchissants représentés sur la figure 13, obtenus en simulant les deux interactions combinées, sont significatifs sur toute la hauteur du pieu, leur maximum se situe à 3,6 m et est égal à $720 \mathrm{kN}, \mathrm{m}$. Ce qui est trés supéricur au cas précédent alor's que l'tnput sismique est le mềme

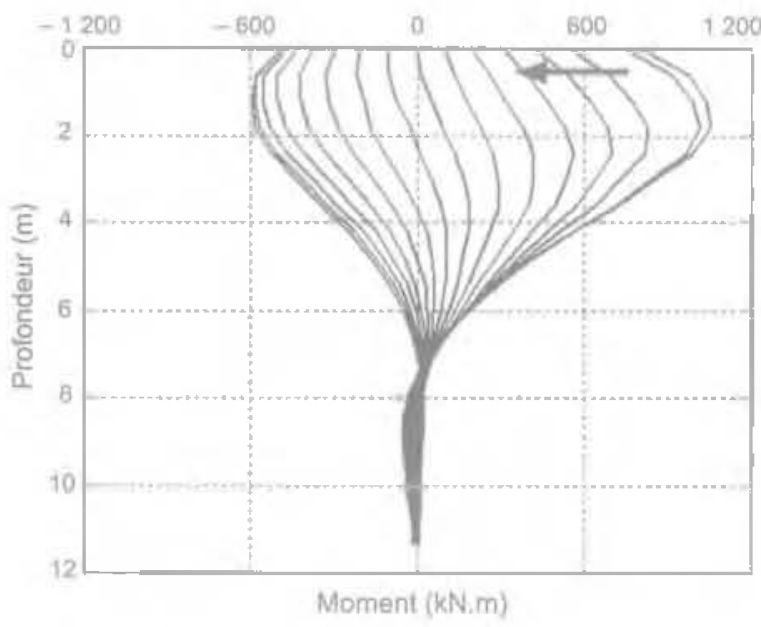

月G 11 Profils du moment fléchissant en différentes séquences du mouvement : essai inertiel. Sequence of bending mament profiles along time at the beginning of an impact test

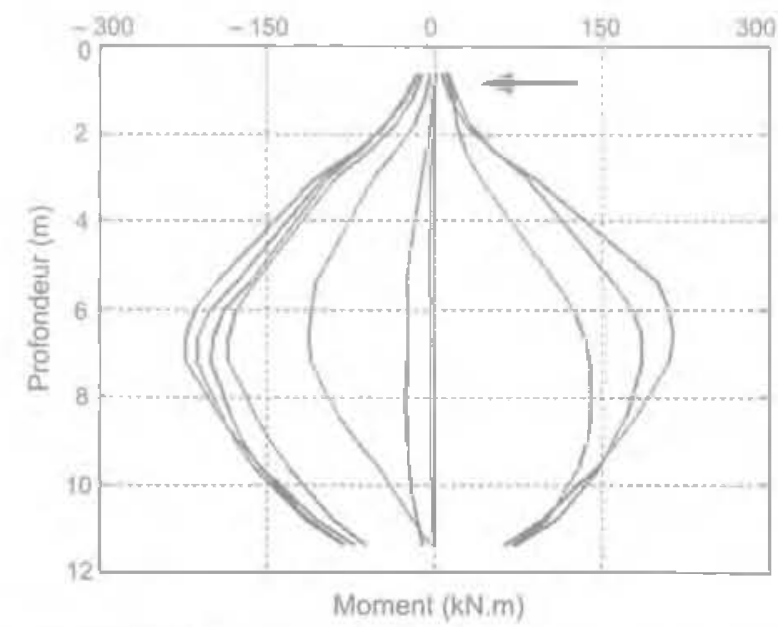

ฆ⿴囗. 12 Profils du moment fléchissant en différentes séquences du mouvement : essai sismique sur pieu ron-équipé du chevêtre. Sequence of bending moment profifes along time during a kinematic test [pile without capl 


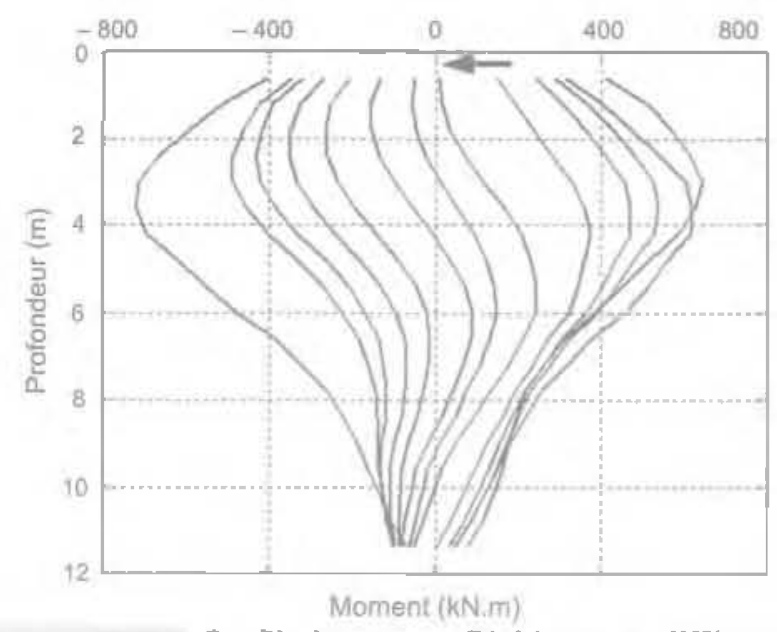

FIG. 13 Profils du moment fléchissant en différentes séguences du rouvement: Essai sismique sur pieu équipé du chevêtre.

Sequence of bending moment profites alone time during a seismic test for combined interactions (pile with cap).

Les courbes enveloppes des profils des moments maximaux positifs pour les trois configurations sont comparées sur ta figure 14. Cette comparaison permet de déduire que

- l'effet inertiel est prédominant au cours cu kremblement de terre pour le pieu équipé d"un chevêtre en tête. Les moments de fortes amplitudes se manifestent dans la zone proche de la tête du pieu;

- le mouvement du sol engendre des défomations du pieu plus importantes dans les couches intermédiaires (situés entre 6 et $10 \mathrm{~m}$ ) en l'absence du chevêtre;

- les essais sous séisme sur pieu équipé ou non équipé du chevêtre montrent l'apparition et la prédominance de l'effet cinématique en pied cu pieu (les courbes sont quasinent confondues à partir ce 8 m).

Des cifficultés expérimentales ont empêche l"abotrtissement à des niveaux de déformations de mêne grandeur pour les essais de chocs et les essais sismiques. Les comparaisons en temes de moments ou de déplacenents maximaux sont limitées, ll serail donc utile de reproduire ces mêtmes essais à un mème niveau de déformation maximale - soit en tête. soit plus bas dans le corps du pieu - pour mieux établir leur

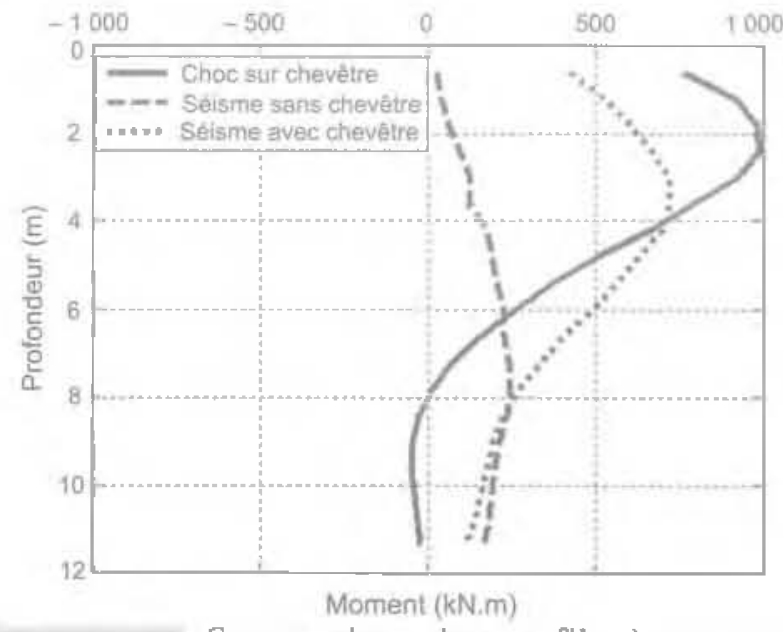

FF. 14

Comparajsor des profils de moments maximaux pour les trois configurations. Comparison of the maximurn bending moment profiles in the three tests configurations. contribution dans l'interaction combinée au cours d'un séisme. Compte teru de la première utilisation du SdS. les essais sismiques sont particuliers à cause de la rigidité du conteneur utilisé. Leffet de réflexion des ondes générées par le tremblement de terre simulé n'a pas été étudié, il done important de reproduire les essais sismiques en reproduisant le mouvement en champ libre en libérant les parois du conteneur.

\section{3}

\section{Modélisation numérique de l'interaction sol-pieu}

Le modèle qui sous-tend le plus souvent les règlements de calcul - que ce soit dans le règlement national (Fascicule 62, 1993) ou les rëglements internationaux (API, 1993; DNV. 1992: PHRI, 1980) - est le modèle du coefficient de réaction qui se traduit par les courbes p-y. Ces courbes expriment la réaction p du sol au dêplacement y du pieu, el]es somt établies expérimentalement à partir des moments fléchissants mesurés par les jauges de déformations collées sur les pieux testés.

L'analyse de l'interaction sol-pieu sous forme de courbes p-y peut servir de base au calage de parametres d'interaction dans un modèle numérique.

Le Laboratoire central des ponts et chaussées a développé le logiciel PILATE-LCPC (PIeux sous charges LATErales) sur cette base (Romagny, 1985 ; Frank. 1999). PILATE-LCPC pernet à partir des courbes p-y statiques expérimentales de calculer lévolution des moments fléchissants, des efforts tranchants, des déplacements et des réactions du sol le long du pleu à partir des courbes p-y statiques expérimentales. Le calcul est basé sur une résolution analytiqua avec un processus de linéarisation des courbes p-y.

Le code développé ici vise à calculer l'évolution des moments, des efforts tranchants, des dëplacements et des réactions du sol te long du pieu en exploitant des courbes $p-y$ expêrimentales, à travers un modèle aux éléments finis ID du pieu. Les interactions sol-pieu sont discrétisées par des éléments d'interface discrets de type Winkler dont les caractëristiques sont extraites des courbes p-y expérimentales. Ce modiele permet en plus d'ajuster des paramètes d'interaction à partir de résultats expérimentaux. Le développement de ce code a d'abord permis de traiter le cas du chargement statique ensuite le cas dymamique.

\section{Code de calcul}

L'équilibre statique d'un pieu de rigidité à la flexion $\mathrm{E}_{\mathrm{p}} \mathrm{I}_{\mathrm{p}}$ soumis à une charge statique $\mathrm{F}$ en tête a pour expression:

$$
P_{s c i l}(y, z)+E_{p} I_{p} \frac{\partial^{4} y}{\partial z^{4}}=F
$$

$P_{\text {sol }}$ est la réaction du sol relative à la profondeur z, y est le déplacement latếra] du pieu dans la direction de la charge F. La masse du chevêtre et le poids du pleu sont négligés.

Pour passer du modèle continu défini par l'équaton (1) à un modèle discret, le pieu est discrétisé par des éléments finis barre deux degrés de liberté : une 

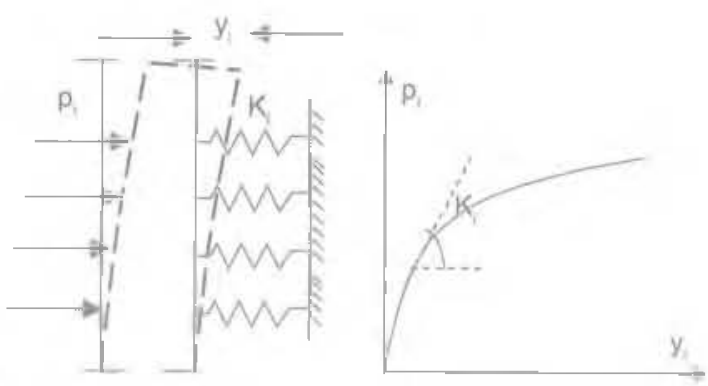

FIS 15

Schématisation du modêle discret de Winkler.

Whinel discret model diagram.

translation ef une rotation à chaque noeud. La rếaction continue du sol est discrétisée par le modéle mécanique de Whkler. Ce modèle néglige la continuité du sol, le sol est assimilé ả un ensemble de ressorts indépendants sur lesquels s"appuie le pieu au cours de son déplacement. La modélisation numérique de l'interaction sol-pieu. par le biais de ce concept, se tradust par la description de cette interaction par une loi de comportement qui relie le deplacement y du pieu à la réaction p du sol. Cette modélisation est décrite par les courbes p-y [Fig. 15].

Dans ce cas, la réaction ponctuelle du sol a pour expression

$$
\mathrm{p}_{1}(y, z)=\mathrm{k}_{\mathrm{r}}
$$

où p est la réaction du sol agissant sur le noud $i ; k$, le coefficient de réaction latérale affecté à ce noud i et $y_{\text {, }}$ le déplacement latéral du pieu corTespondant.

Le coefficient $k$ est ume grandeur dinteraction solpieu. Il qualifie la raideur du sol vis-à-vis du déplacement latéral du pieu. Compte tenu de la non-linéarité du comportement du sol, il est souvent dépendant du dêplacement y du pieu, ce n'est donc pas un parametre intrinsèque du sol.

A partir de ces hypothèses, un algorithme basé sur une résolution en déplacement a été développé pour résoudre l'équation (1). Chenal (2007) décrit l'élaboration de cet outil de calcul en détails ainsj que la combinaison de deux algorithmes: discrétisation spatiale par la méthode des éléments finis et linéarisation par la méthode de Newton-Raphson. Un processus de chargement incrémental est introduit pour assurer la convergence de l'algorithme (Mestat, 1988).

La validation du modẻle statique, développé sous MATLAB, a été effectuée selon deux approches :

- la comparaison des résultats avec ceux d’une résolution analytique:

- la comparaison avec des rẻsultats de PILATE-LCPC fondés sur les mêmes résultats expérimentaux.

\section{2}

\section{Validation du modèle statique par une solution analytique}

Frank (1999) a présenté une solution analyt que, dans le cas d'un sol homogène et élastique de module $\mathrm{E}_{\text {, }}$ le pieu étant soumis à un chargement latéral statique. La solution en déplacenent de l'équation (1) est donnée par la relation (3). Le moment fléchissant. qui correspond à la deuxième dérivèe du déplacement. est donné par l'équation (4) :

$$
\begin{aligned}
& y(z)=e^{z / 1},\left[a_{1} \cos \frac{z}{1_{0}}+a_{2} \sin \frac{z}{1_{0}}\right]+e^{-z / I_{0}}\left[a_{3} \cos \frac{z}{I_{0}}+a_{4} \sin \frac{z}{I_{0}}\right]_{(3)} \\
& \frac{M}{E_{p} I_{P}}=y^{\prime \prime}(z)=\frac{2 e^{z / 1}}{1_{0}^{2}}\left[-a_{1} \sin \frac{z}{1_{0}}+a_{2} \cos \frac{z}{l_{0}}\right]+\frac{2 e^{-z / l_{0}}}{l_{0}^{2}}
\end{aligned}
$$$$
\left.\operatorname{la}_{3} \sin \frac{2}{\mathrm{I}_{0}}-\mathrm{a}_{4} \cos \frac{\mathrm{z}}{\mathrm{I}_{0}}\right]
$$

Les termes $a_{1}, a_{2}, a_{3}, a_{4}$ sont des constantes d'intégration qui peuvent être déterminées à partir des conditions aux limites. $I_{a}$ est la longueur de transfert du pieu donnée en fonction du module de sol E, du module du piau $E_{\text {p }}$ et de l'inertie de sa section par rappont à son axe transwersal i

$$
I_{0}=\sqrt[3]{\frac{4 E_{\mu} I_{p}}{E_{y}}}
$$

Le module de réaction $E_{\text {correspond au coefficient }}$ de reaction $k(y, z)$ pour un diamètre $B$ donné du pieu:

$$
\mathrm{E}_{\mathrm{s}}=k(y, z) \mathrm{B}
$$

Pour l"application numérique, on a pris une des valeurs du module de réaction proposées par Poulos (1971) (Tableau V). Ces valeurs moyennes ont été déterminées à partir d"essais sur des pieux réels enfouis dans clu sable (Broms, 1964).

Les figures 16 et 17 représentent les résultats analytiques obtenus par le calcul du déplacement et du profil de moments flechissants à partir des équations (3) et (4). comparés à ceux obtenus par le modèle numérique (Chenaf, 2007). Ces deux figures montrent que les résultats des deux calculs sont comparables. L'Ecart qui apparaît au niveau du moment maximum $(a ̀) 1,8 \mathrm{~m}$ de profondeur) n'excède pas $10 \%$. Notons que l'estimation de la propagation cle l'erreur de la mesure de moments - estimée à $3,7 \%$ - engendre ure incertitucle de $0.5 \%$ sur le calcul du déplacement du pieu y et une incertifude de $15,5 \%$ sur la réaction du sol p à cette profondeur (Chenaf, 2007). Rappelons à cet effet que co sont les courbes p-y qui sont assujetties au processus d'ajustement par le code que nous avons établi, d'où la justification d'un tel écart avec les mesures.

Validation du modèle par comparaison avec des résultats expérimentaux et avec un calcul avec le logiciel PILATE-LCPC

Des mesures issues d'essai de chargement stalique monotone (Rosquoët, 2004) ont été utilisées pour effectuer cette validation. Elles ont permis d'ajuster sur ces coupbes p-y expérinentales les caractéristiques des ressorts de winkler n. Les courbes p-y de surface ont été ajustées par des fonctions de la forme $\mathrm{p}=\mathrm{Ay}$ (PHRI, 1980: Mezazigh, 1995). Les courbes des couches profondes ne présentant pas de fortes non-linéarités, la relation p-y a êté ajustée par des droites. Un exemple de chaque mode d'ajustement est donné sur les figu-

sableaur Valeur moyenne du module d'Young $E_{5}$ pour différentes tensités de sable.

Means watues of the soil young modulus $E_{s}$ of sand as function of its density.

Densité du sable Intervalle de la valeur Es $\left(\mathrm{kg} / \mathrm{m}^{2}\right)$

$\begin{array}{lc}\text { Läche } & 91400-210920 \\ \text { Moyen } & 210920-421840 \\ \text { Dense } & 421840-984300\end{array}$




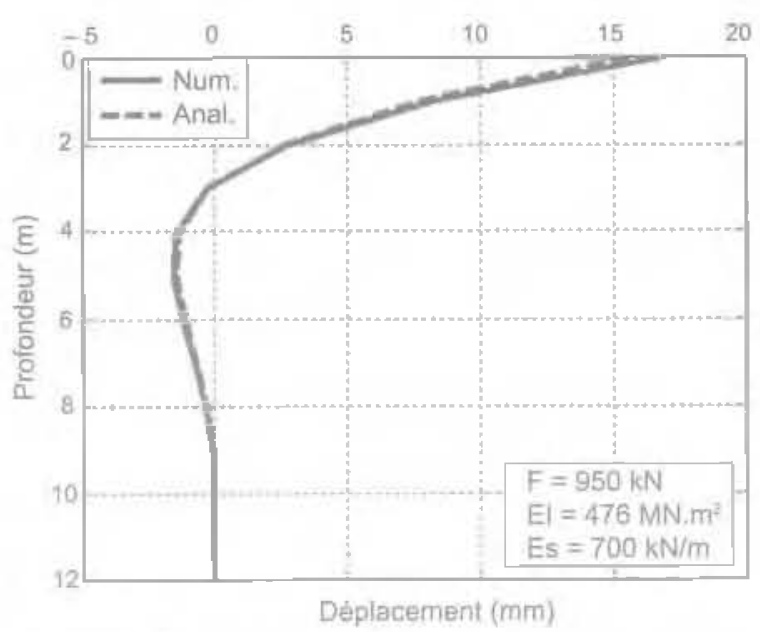

Fe 16 Comparaison du calcul analytique et du code EF 1 D en statique : profil du déplacement,

Comparison of rumerical model to analyticat solution : displacement profile.

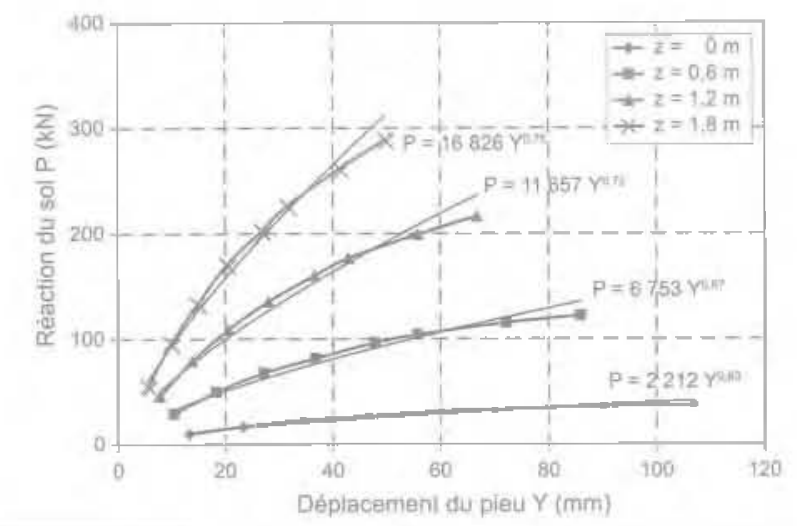

FG. 18 Ajustement de type puissance des courbes expérimentales $\mathrm{p}-\mathrm{y}$.

Expermental p-y fitting : power law fitting.

res 18 et 19 : les lignes marquóes comespondent aux courbes experinentales et les lignes continues correspondent aux courbes ajustées.

Ces mërnes courbes experinentales ont été introduites dans le logiciel PILATE-LCPC pour calculer le moment, l'effort tranchant, le déplacement du pieu et la réaction du sol.

La figure 20 représente les courbes expérimentales, les courbes obtenues par PILATE-LCPC, confrontées aux courbes obterues par notre code. Ces courbes montrent une meilleure concordance entre les résultats expérimentaux et la simulation effectuée avec notre code EF 1D qu'avec le logiciel PILATE-LCPC. Rosquoët (2004) admet d'ailleurs que PILATE-LCPC sous-estime légèrement les résultats, il a remarqué que ce phénomène est amplifié avec l"accroissement de la charge F appliquée en tête du pieu. [l a montré néanmoins que la différence reste inférieure a $10 \%$.

Notons qu"en plus du gain de précisions que procure notre code, il présente l'avantage de la flexibilité d'application de ta charge exterieure et du choix du nombre d’appuis. PILATE-LCPC ne permet de l'appliquer qu'en tếte et le nombre de points de calcul dans le sol est limité ì 20.

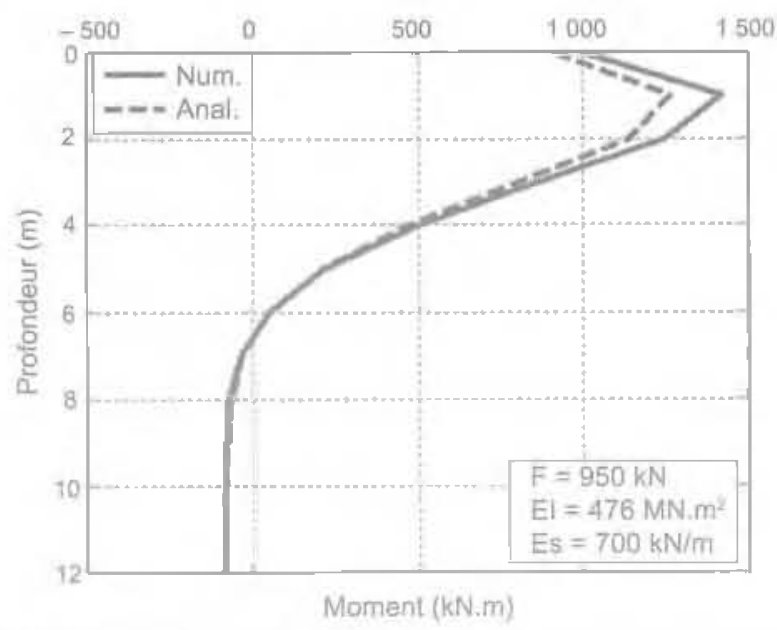

m6. 17 Comparaison du calcul analytique et du code 1D en statique: profil di moment flechissant.

Comparison of numerical model to analytical solution : bending moment profile.

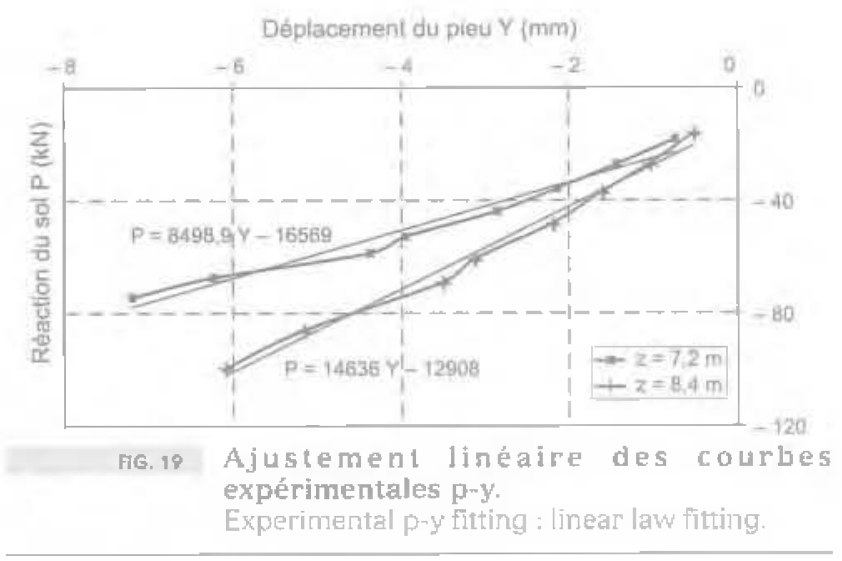

A partir de ces deux comparaisons, on a considéré le code EF $1 \mathrm{D}$ comme validé pour la modelisation de l'interaction dans le domaine non linéaire sous charge statique et on a pu entreprendre la modélisation dans le domaine dynamique.

\section{4}

\section{Modélisation numérique \\ de l'interaction inertielle et cinématique}

L'algorithme statique validẻ a été adapté au calcu] et l'analyse de l'interaction non linéaire sous charge dynamique appliquée en tête. L'équilibre dynamique en flexion unidimensionnelle du modèle cortinu du pieu s'écrit dans ce cas:

$\mu_{p} \frac{\partial^{2} y}{\partial t^{2}}+c_{p} \frac{\partial y}{\partial t}+p_{s o l}\left(y, \frac{\partial y}{\partial t}, \frac{\partial^{2} y}{\partial t^{2}}, z, t\right)+E_{p} I_{p} \frac{\partial^{4} y}{\partial z^{4}}=F(z, t)(7)$

où $F(z, t)$ est la force extérieure appliquée en tête : $p_{\text {sol }}\left(y, \frac{\partial y}{\partial t}, \frac{\partial^{2} y}{\partial t^{2}}, z, t\right)$, la force de réaction iransversale $d u$ 

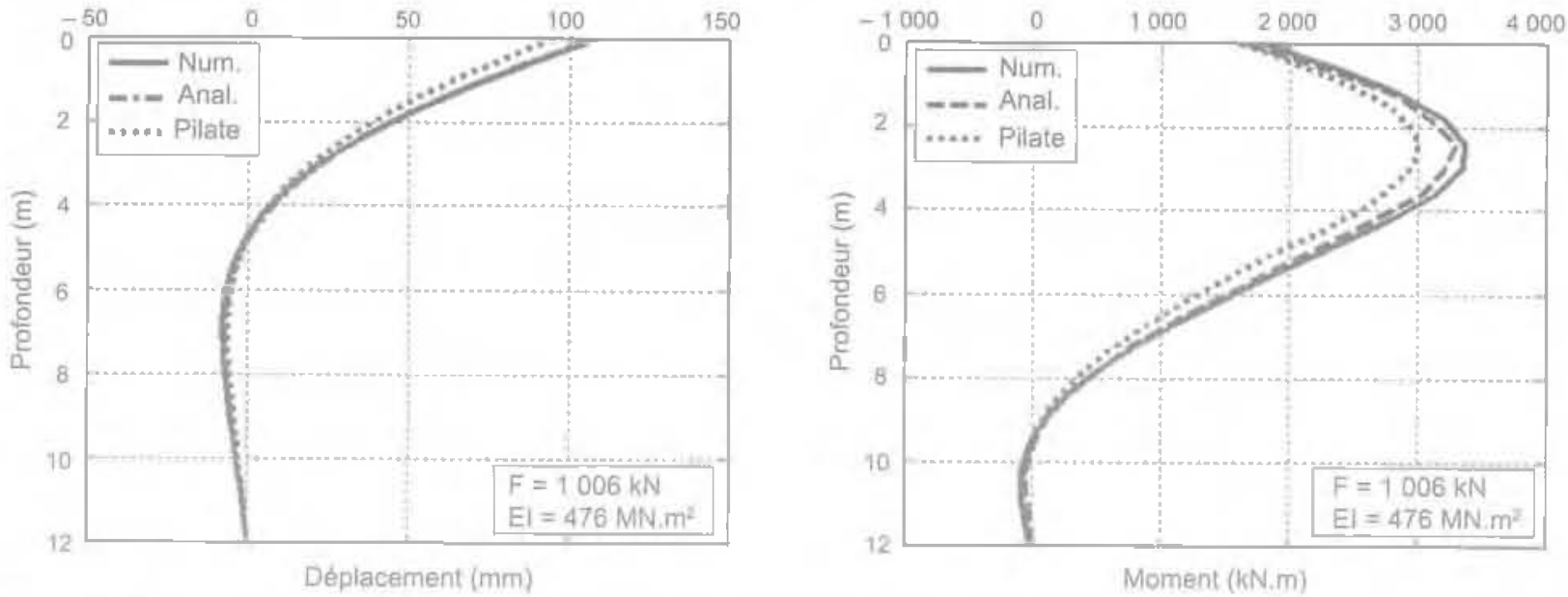

Fis. 20 Comparaison des profïs des déplacements et des moments simulés et mesurés. Comparison of computed and measured displacements and bending moments profiles.

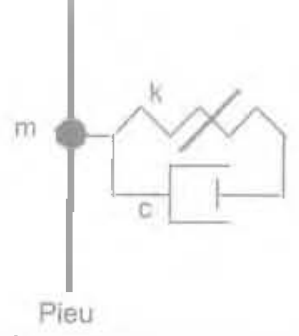

FG. 21 Schéma de l'élément d'interface de Winkler pour une charge dynamique.

Basic dalagram of the dynamic Winkler beam interface elements.

sol: $E_{p} I_{F} \frac{\partial^{4} y}{\partial z^{+}}$, la force d'équilibre de la densité de chargement dans la direction de la translation due au moment de flexion du pieu: $c_{1} \frac{\partial y}{\partial t}$, la force d'amortissement interne du pieu et $\mu_{n} \frac{\partial^{2} y}{\partial t^{2}}$, la force d'inertie de translation du pieu qui comprend la masse du chevêtre.

La réaction p du sol est déconpousee en trois forces une force élastique dans le ressort, équivalente à la réaction statique développée dans le paragraphe pré-

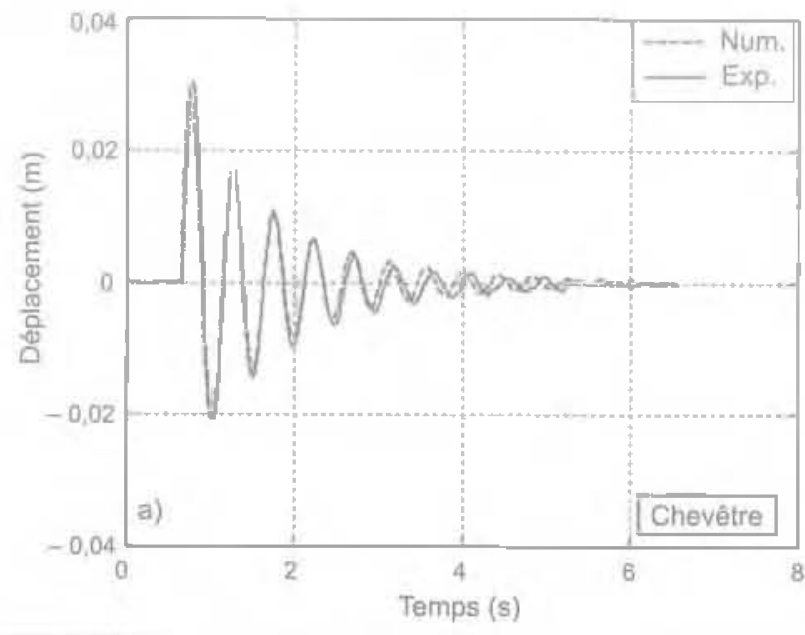

cédent, une force d"amortissement développée dans un amortisseur" et une fol"ce d'inertie développée dans une masse. Ce modèle, attribué à chaque appui de Winkler. est schématisé sur la figure 21.

Lalgorithme tempore] HHT (Hilbert-Hugues-Taylor, 1977) a été implémenté dans le code statique pour la résolution de l'équilibre dynamique du systême superstructure-sol-pieu. Deux cas de chargements dynamiques ont été traités : le choc en tête et le séisme. L'algorithme est adapté au chargement impulsionnel en tête par l'introduction d'une condition de vitesse initiale non-nulle calculée à partì de la quantité de mouvement cédée au chevêtre par l'impact. Le chargement sismique est introduit par le biais d'une force d'inertie relative aux mouvements du sol qui entoure le pieu en chaque appui de Winkler.

Ce code a été utilisé pour ajuster des modèles d'interface fonciés sur le schéma de la figure 2"l qui se composent d'un élément de réaction élastique statique, d"un élément d'amortissement et d'un élément de masse.

Les éléments d'interaction êlastiques ont étéf fondés sur des lois d’interaction de la littérature pout" modé liser un ressort non linéaire (PHRI, 1980; API, 1993: Carter, 1984]. La force d'amortissement a été estimée par une loi classique de calcul de propagation donde dans un milieu continu et homogène (Gazetas et Dobry,

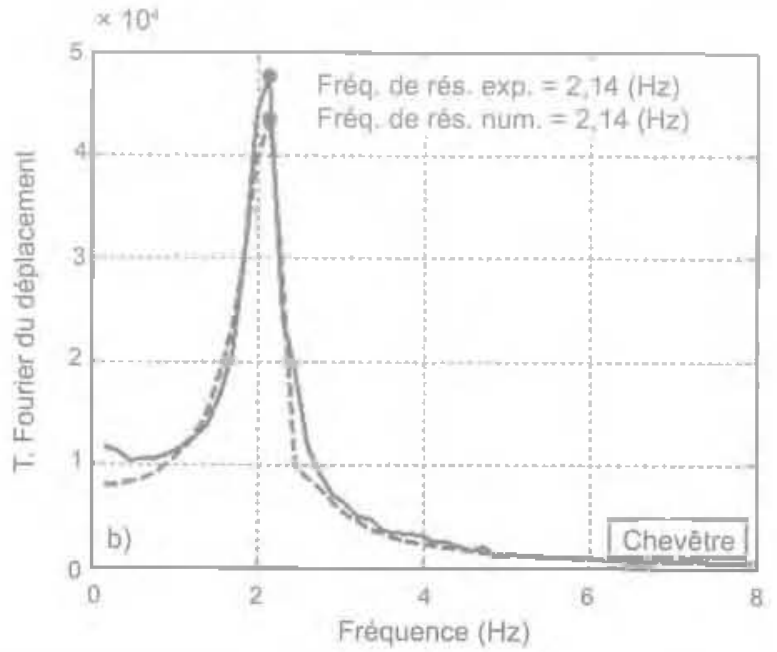

FIG. 29 Comparaison des courbes expérimentales et des courbes simulées : déplacement du chevêtre : a) domaỉne temporel; b] domaine fréquentiel.

Comparison of experimental and numerical pile cap displacements : (a) in time domain. (b) in the freguency domain. 

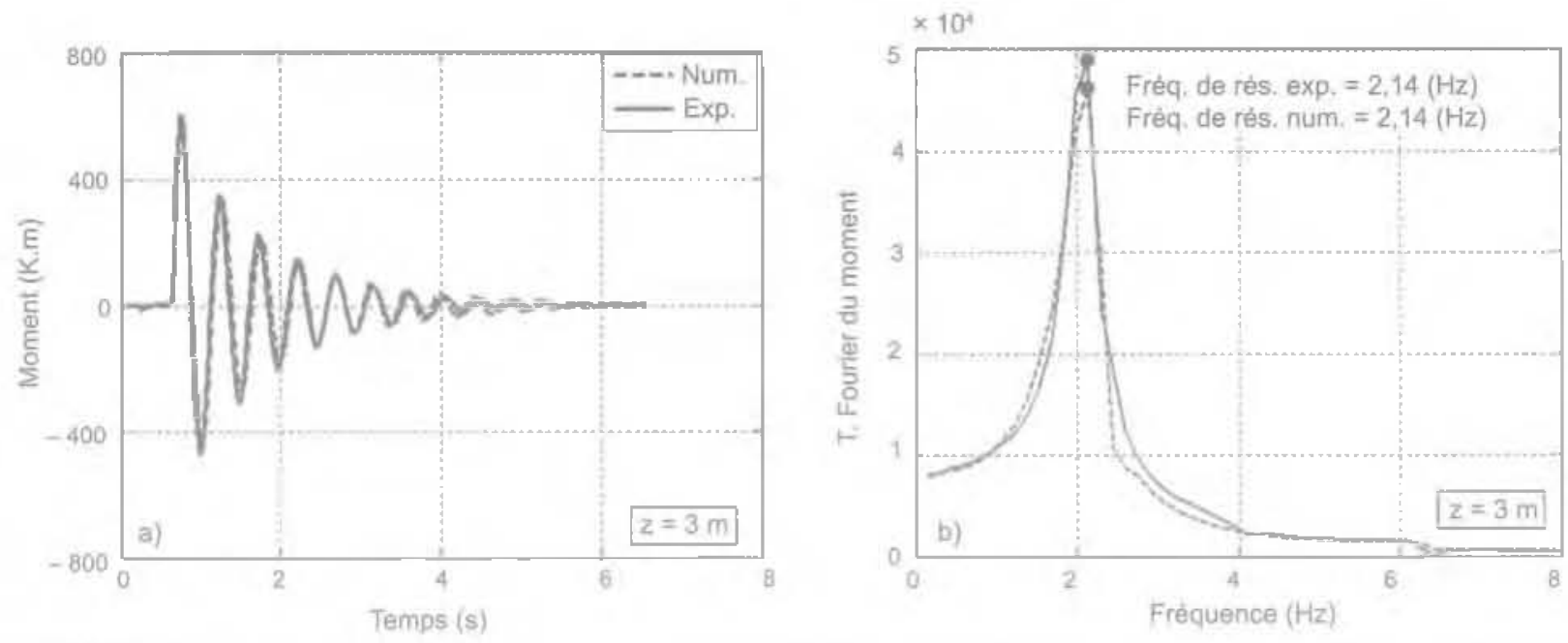

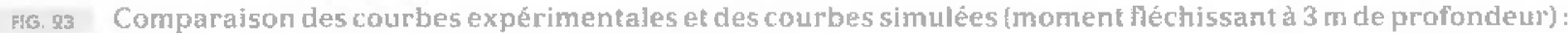
a) domaine temporel; b) domaine fréquentiel. Comparison of experimental and mumerical bending moment $3 \mathrm{~m}$ deep : (a) in time domain, Ib) in the frequency domain.

1984). Le paramètre de masse a été pris équivalent à la masse d'un cylindre de sol déplacé dont le diamétre est proportionnel à celui du pieu.

Les courbes présentées sur les figures 22 et 23 montrent un exemple d'ajustement fondé sur une loi puis-

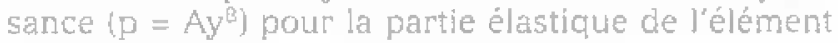
d'interaction. Les courbes calculées sont comparables aux courbes mesurées. Les fréquences de résonance et les amortissements sont identiques tant sur le déplacement du chevêtre que sur la courbe de moment. Un écart apparaît cependant entre les courbes temporelles au-delá de 3 s. signe d'un raidissement du modèle numérique en fonction du temps.

Trois lois décrivant la raideur ont étë implémentẻes et leurs paramètres ajustés sur les mesures. Ces trois lois ne montrent pas de performance relative, elies ont cependant permis d'ajuster des paramètres en appliquan! des coefficients d'ajustenent sur les paramètres initiaux déduits des mesures. Dans le cas du chargenent sismique, une loi linéaire a été implémentẻe èt ajustée.

Ces lois d'interaction ajustées n'ont été testées que pour une série d'essais. En revanche, la stabilité et la convergence du code ont éte vérifiées et validées.

\section{Conclusion}

Par une approché tant expérimentale que numérque, on a pu entreprendre l'étude détaillée de deux for mes d'interaction quj régissent l'interaction entre le sol et un pieu de fondation lors d'un sếisme : l'interaction inertielle et l’interaction cinématique.

La modélisation physique à échelle réduite en centrifugeuse a permis une observation expérimentale indépendante de ces deux interactions qui se superposent normalement lors d'un séisme. Linteraction inertielle seule a été mise en évidence par des essais de chocs appliqués en tête du pieu ä l'aide d'ur impacteur électromagnétique. Linteraction cinématique a été isolée en réalisant un essai sismigue avec le nouveau simulateur de séisme du LCPC en supprimant le chevê. tre massif́ en tête du pieu. Enfin, un essat sous séisme mais avec le chevêtre remis en tête du pieu a permis d'observer les deux interactions combinées.
Il convient de rappeler ici que le conteneur d'essai pour les essais sismiques était un contene-ur rigide. De ce fait, le champ d'accélération clans le conteneur n'était pas conforme à ce qu'il peut être dans un demi-espace infini. De plus, on peut penser que les ondes générées dans le sol par les interactions ont pu se réfléchir sur les parois rigides (principalement dans la clirection longitudinale de l'ébranlement sismiguel. Cela a eu donc des conséquences en termes d'amplitudes des efforts et des déplacements le long du pieu, on peut: aussi penser que cela n'invalide pas la relation entre les déplacements et les réactions di sol sur le pieu qui peuvent étre déduites des profils de moment dans le pieu. Il est bien sûr nécessaire de reprendre l'ensemble des essais sismiques - avec et sans chevểtre - en utilisant un conteneur à parois déformables de manièe se rappmocher des profils d'accélération de champ libre. Cela permettra de vérifier les relations entre le déplacement du pieu el la réaction du sol $(p-y)$ et rendre plus réaliste la répartition des efforts en profondeur.

Pour exploiter ces données expérimentales, un code de calcul par éléments finis $1 \mathrm{D}$ a été développé sur la base du modèle de la poutle de Winkler sur appuis discrets non linéaires. Il a d'abord été validé dans le domaine staticue et s'avère plus précis et pius souple - dans le cas testé - cue l'outil disponible fondé Str une solution analytique. Étendu au domaine dynamique pour un calcul de réponse dans te domaine temporel, il constitue un banc de developpement de modeles d'interface par ajustement de paramètres sur des données expêrimentales. L'application de cel outil aux données issues des trois sêries d'expérimentation présentées a permis la mise au point d'une première famille de modèles d'interface simples traduisant bien les trois configurations d'essais. Ceci ne constitue pas une validation de ces éléments d’interface : il convient de rẻaliser de nouvelles séries d'essais en modifiant différents paramètres lpar exemple : diverses configurations d'essaj sismiques - fonctions hamoniques et non harmoniques - et divers chevêtres en tête dans le cas du chargement impulsionnel'! pour vérifier que les modèles présentent une certaine robustesse. Au cours de cette démarche. il est probable que de nouvelles familles de modèles d'interface, peut-être un peu plus sophistiqués, s'avéreront nécessaires. 


\section{Bibliographie}

API (American Petroleurn Institute) - Designing and constructing fixed offshore platforms. RP2A-LRFD. Section G. 1993 p. 6477 .

Baguelin F. Frank R., Guegan Y - CalcuE sur ordinateur des pieux sollicités hort. zontalement ou subissant des poussées parasites. Bulletin des laboratoires des ponts el chaussées, 84, 1976, p. 113-120.

Barghouti A.F. - Pile response to seismic waves. PhD dissertation. University of Wisconsin, Mardison, 1984

Bonab H.M. - Modelisation physique et numérique d'un pieu isolé dans du sable soumis à un impact latéral en tête. Thèse de doctorat. université de Caen. 2003 228 p.

Boulanger R.W Currus CJ Kuter B. Wilson D.W. Abghari A. - Seismic soil pile-structure interaction experirnenes and analyses. Journal of Geotechnical and Geoenwronmenta! Engineering. vol. $12 \mathrm{~s}, n^{\circ} 9,1999$, p. $750-750$.

Broms B.B. - Lateral resistance piles on cohesionless soils. American societ. of civil angineering journal, $5 \mathrm{M} 3,1964$ p. $123-156$

Carter D.P. - A non-linear soil model for predicting lateral pile response. Rep $n^{\circ}$ 359. Civil Engineering Dept. Liniver sity of Auckland. New Zealand, 1984.

Chakraborty S. - Dynanic laterad loading testing of deep foundation groups. Pho thesis, University of Auburn, 2000 $286 \mathrm{p}$

Chenaf N. - Interaction inertielfe et inte raction cinématique sol-pieu. Thèse de Doctorat, É Eole centrale de Nantes-Université de Nantes, $2007,330 \mathrm{p}$

Derkx. F. Thorel L... Chazelas .T.-L., Escoftier S., Rault G., Buttigieg S. - Dynamic tests and simulation of earthguake in the LCPC's centrifuge. 6th InternatioMal Conference on Physical Modelling in Geotechrics, 4-6 August. Hong-Kong. 2006a, p. 181-186

Derkx F., Thorel L., Chazelas J.-L., Perdriat I. Escoffier 5. Rault G. Buttigieg S. Cottineaı L. - M. - Simulation de séjismes sous macro-glavité. Revite Essais industriels, $\pi^{\circ} 39,2006$, p. 12-21

DNV (Det Norske Veritas! - Rules for the design construction and inspection of offishore structures. Appendix F : Fourdations, $1992,5 \mathrm{p}$.

Dobry R. O'Rourke M.3. - Disetussion on 'Seismic l'esponse of end-bearing piles by Flores-Berrones R. and Whitman R.V. Journal of Geotechnical Engineering Division, ASCE, n० 109, 1983.

El Naggar M.H. Bentley K.J. - Dynamic analysis of laterally loaded piles and dyramic p-y curves. Canadian Gentech. gical Joumal, vol. $37.7^{\circ}$ 6. 2000, p. 1166. 1182

El Naggar M.H. - Interpretation of latera STATNAMIC load test results. Geotechnical testing journad, wol. 21, 1398, p. 169 179

El Naggar H., Navak M. - Non-linear analysis for dynamic lateral pile response. Soil Dynamics and Earthquake Engineering. vol. 11, 1996, p. 233-244.
Fascicule na 62. Titre V - Regles technigues de conception el calcul des fondations des ourrages de genie civil. Cahier des clavses techriquers generates apolicables aux marchés publics de travaux. Minis tère de l"Équipement, du Logement et des Irarsports, 1993, $182 \mathrm{p}$

Flores-Berrones R., Whitman R.V - Seis mic response of end-bearing piles. four nat of Geotechnical Engineering. ASCE. val. $108, n^{\circ} 4$. 1982 p. $554-569$.

Frank F. - Calcul des fondations superficiel Jes et profondes. Editions Technique de I'Ingénleur et Presse des Ponts et Chaus sées, 1999, 141 p.

Gazetas $G$ - Seismic response of end-bea ring single piles. Soil Dymamics and Eatthowake Engineering, vol 3, no 2 1984 p. $82-93$

Gazetas G. Dobry R. - Horizontal response of piles in layered soils. Journal of Geotechnical Engineering. ASCE, vol. 110 , 798 s $20-40$.

Grundhoff T. Latotzke J., Laue J. - Investigations of vertical piles under horizonta. impact. Centrifuge 98. 1998, 5.569-574.

Hilbert H.M., Hughes T.J.R. Taylor R.L. - Improved numerical dissipation for ime integration algorithms in structura dynamics. Earthquake Engineering and Structural Dynahncs Journal, vot. 5, 1977. D. $283-292$

Kaymia A.M., Katusel E. - Dynamic beha. viour of pile groups. 2nd Intermationa! Conference on Numetical Methods it Offishore Pling. Austin. Texas, 1982

Maheshwari B. Truman K.Z., El Naggar M.H. Gould P.L.. - Three-dimensiona finite element moninear dynamic ana Iysis of pile groups for lateral transient seisiric excitations. Canadian Geotechnical Journa!', vol. 41, 2004a, p. 118-133.

Mahshwari B.K., Truman K.Z. El Naggar M.H. Gould P.L. - Three-dimensional nonlinear analysis for seismic soll-pile. 5tructure interaction. Soil Dynamics and Earthkaque Engineering Journat, vol. 24. 2004b, ip 343-356.

Makis N., Gázetas G. - Dynamic pile-sojl. pile interaction. Part IT : Lateral and seis. mic response. Earthquake Engheering Structural Dytamics. vol, 21, n 2, 1992 p. $145-162$

Margson E. - Ple bending during earthcuake. Lecture, 6 March 1975 , ASCE-UC Bervey Seminar on Dosign Construction and Performance of Deep Foundatons. 1975

Maklock H., Foo S.H., Bryarat L. L. - Simulation of laterai pille behaviour. 6th forter national Conferemce on Soll Herthanics and Foundarion Engineering. Montréal. wo. $2,1978,0.275-279$

Mestat P. - Les méthodes de résolution en comportement non lineaire. Theorie et apalication. Papport FAED \& 05.70 .7 Laboratoire Central des Ponts et Chaussèes (litterature grise GEOGO1310! 1988

Meymond P.s. - Shaking table scale mudel tests of norlinear soll-pile-structure inte raction in soff clay. Ph.D. Thesis. University of California, Berkley, 1998.
Mezazigh S. - Etude expërmentale de pieux chärgés latératement : proximité d"un falus et effet de groupe. Thèse de doctorat, université de Nantes, 1995 , 266 p.

Myonakis G. Nikotaou A. Gazetas G. Soil-Pile-Bridge seismic interaction: Kingmatic and inertial effects Part I: Soff soil. Earthquake Engineering and Strut tural Dynamics, vol. $26,1997,19,337-359$

Nikolaou' S., Mytonakis G., Gazetas G. Tezon T - Kinematic pile bending during earthquakes: analysis and field measure. ments. Geotechnigue vol. 51, $n^{2} 5,2001$. p. $425-440$.

Nogami T. - Soil pile Interaction motel for earthquake response analysis of offs. hore pile foundations. 2nd internations conference on recent advances in Geotechnical Earthquake Enginezring and Soil Dynamies, vol. 3. St Louis, 1997

Novak M., Aboul-Ella F - Impedance functions of piles in lavered media. Jourtat of Enginesring Mechanics. ASCE. wol. 104 $0^{\circ}$ EMF 1978

Novak M. - Dynamic stiffness and damping of piles. Canadian Geotechnical Journai vol. 11, 1974, D. 574-598.

PHRI (Port and Harbour Research Institutel - Tochnical standards for porr and harbour facilities in Japan. Bureau of ports and harbours. Ports and Harbour Research Institute. Ministry of Iransport. $1980.317 \mathrm{p}$.

Poulos H. - Behaviour of laterally loaded piles: 1-single pile. Joumal of Geotechnical Engineering, ASCE, vol. 101, n $5 \mathrm{M} 5$, $1971,733-751$

Remaud D. - Pietdx sous charges laterales etude experimentale de l'effet de groume. Thèse de doctorat, Lniversité de Nantes. 1999 , $328 \mathrm{p}$

Romagny J - C. - Programme de caloul d'un pien isolé soumis à des efforts de flexion

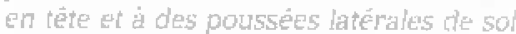
Notice d'utilisationt. LCPC. Partis $1 \pi$ version J.-L. Brangratz, R. Frank, M. Kutniak. 1985,69 p

Rosquoèt F. - Piewx sous charge latérale cycligue. These de doctorat, Fcole censrale de Nantes-Universite de Nantes. 2004, $305 \mathrm{p}$.

Shahrour I. Benzenatj I, Ousta R. - Comportement des pieux sous chargement lateral đyparnigue : anglyse bibliogra. phicue. Projet national Renforcenent des sols par' micropleux. Papport interme N Fo/93/04, 1994.

Souloumiac R. - Methode simplifiee de catcul des pjeux en zones sismiques. Annales de Jinstitut technigue du battment et des travalu publics, $h^{\circ}+11,1986$. p. 61-87.

Tokimatsu K, Suzuki H., Sato M. - Effects of inertial and kinematic interaction on seismic behaviour of pile with ember. ded foundation. Sôt Dynamics and Earthkaque Engineering, vol. 25, 2005, D. $753-76$ ?

Wilson S.W. - Soil-pile-structure Interaction in liquefying sand and soft clay. Ph.D. Thesis. University of California, Davis. $1998,173 \mathrm{p}$. 